\title{
The AMIGA project
}

\section{Optical characterization of the CIG catalog $\star, \star \star$}

\author{
L. Verdes-Montenegro ${ }^{1}$, J. Sulentic ${ }^{2}$, U. Lisenfeld ${ }^{1,3}$, S. Leon ${ }^{1}$, D. Espada ${ }^{1}$, E. Garcia ${ }^{1}$, J. Sabater ${ }^{1}$, and S. Verley ${ }^{1,4}$ \\ 1 Instituto de Astrofísica de Andalucía, CSIC, Apdo. 3004, 18080 Granada, Spain \\ e-mail: lourdes@iaa.es \\ 2 Department of Astronomy, Univ. of Alabama, Tuscaloosa, USA \\ 3 Dept. Física Teórica y del Cosmos, Universidad de Granada, Spain \\ ${ }^{4}$ LERMA - Observatoire de Paris, 61 Av. de l'Observatoire, 75014 Paris, France
}

Received 29 October 2004 / Accepted 16 February 2005

\begin{abstract}
The AMIGA project (Analysis of the Interstellar Medium of Isolated Galaxies) is compiling a multiwavelength database of isolated galaxies that includes optical (B and $\mathrm{H} \alpha$ ), infrared (FIR and NIR) and radio (continuum plus HI and $\mathrm{CO}$ lines) properties. It involves a refinement of the pioneering Catalog of Isolated Galaxies. This paper is the first in a series and begins with analysis of the global properties of the nearly redshift-complete CIG with emphasis on the Optical Luminosity Function (OLF) which we compare with other recent estimates of the OLF for a variety of environments. The CIG redshift distribution for $n=956$ galaxies re-enforces the evidence for a bimodal structure seen earlier in smaller samples. The peaks at redshift near 1500 and $6000 \mathrm{~km} \mathrm{~s}^{-1}$, corresponding respectively to galaxies in the local supercluster and those in more distant large-scale components (particularly Perseus-Pisces). The two peaks in the redshift distribution are superimposed on 50\% or more of the sample that is distributed in a much more homogeneous way. The CIG probably represents the most homogeneous local field example that has ever been compiled. Our derivation of the CIG OLF is consistent with other studies of the OLF for lower density environments. This comparison via the Schechter parameter formalization shows that: 1) $M^{*}$ increases with galaxy surface density on the sky and 2) $\alpha$ shows a weaker tendency to do the same. The CIG represents the largest and most complete foundation for studies of isolated galaxies and is likely as close as we can come to a field sample.
\end{abstract}

Key words. galaxies: evolution - galaxies: interactions - galaxies: luminosity function, mass function - surveys

\section{Introduction}

The evolutionary history of galaxies is thought to be strongly conditioned by the environment. Evidence has emerged for interaction-induced emission enhancements (e.g. Sulentic 1976; Larson \& Tinsley 1978; Joseph \& Wright 1985; Bushouse 1987; Xu \& Sulentic 1991, hereafter XS91) and interaction-driven secular evolutionary effects (e.g. Moore et al. 1996; Verdes-Montenegro et al. 2001) in galaxies that are members of binaries and dense groups. The observational evidence is sometimes weak or unclear. Part of the difficulty lies in the confusion between the roles of one-on-one interactions vs.

* This research has made use of the NASA/IPAC Extragalactic Database (NED) which is operated by the Jet Propulsion Laboratory, California Institute of Technology, under contract with the National Aeronautics and Space Administration.

$\star \star$ Full Tables 1-3 are available in electronic form at http://www.iaa.csic.es/AMIGA.html or from the CDS via anonymous ftp to cdsarc.u-strasbg.fr $(130.79 .128 .5)$ or via http://cdsweb.u-strasbg.fr/cgi-bin/qcat?]/A+A/436/443 more general correlations with average galaxy environmental density. Many of the uncertainties, both of the amplitude of enhancements and the connection between environment and parameters, reflect a lack of suitable control samples to which interacting sample properties can be compared. Ideally this would involve samples of isolated galaxies. Samples of isolated pairs and compact groups provide the parameters to quantify effects of 2 and $n$ body interactions. Isolated galaxy samples should provide the baseline for interpreting the strength and properties of interaction-induced effects. Awareness of these two effects (one-on-one vs. local overdensity) is important in compiling isolated galaxy samples.

The most common reference or control samples found in the literature can be described as either "field" or "normal". The former refer to the most isolated galaxies while the latter refer to galaxies which show none of the generally accepted signs of interaction-induced activity. A field sample (e.g. Kennicutt \& Kent 1983) might include any galaxy not belonging to a cluster, so galaxies in pairs, triplets and loose/compact groups would 
not necessarily be excluded. Normal galaxy samples would be defined in terms of specific parameters such as HI content (Boselli et al. 2001) or a specified level of nuclear activity. Study of a selected quantity as a function of the environment is then one way to quantify the level of environmentally induced activity.

The alternative approach involves sample selection using an isolation criterion. In the case of isolated binaries this likely reduces the interaction equation to the effects of one-on-one encounters. Studies of isolated galaxies usually involve from $10 \mathrm{~s}$ to 100-200 objects (e.g. Huchra \& Thuan 1977; Vettolani et al. 1986; Marquez \& Moles 1999; Marquez et al. 2000; Colbert et al. 2001; Pisano et al. 2002; Varela et al. 2004). The largest samples of isolated galaxies in the literature involve, in most cases, monochromatic observations of subsamples from the Catalog of Isolated Galaxies (CIG: Karachentseva 1973, also referred as K73 in SIMBAD and KIG in NED databases; see Sect. 2) (Adams et al. 1980; Haynes \& Giovanelli 1980; Sulentic 1989; Young et al. 1986; XS91; Hernandez-Toledo et al. 1999; Perea et al. 1997; Sauty et al. 2003).

Previous work suggests that small samples of isolated galaxies have limited statistical value. Ideally we seek a sample large enough to isolate a significant population of the most isolated galaxies. This motivated us to use the CIG as the basis for a large, well-defined and statistically significant multiwavelength database that can serve as a comparison template for the study of galaxies in denser environments. CIG galaxies were selected to be free of equal mass perturbers but hierarchical pairs and groups could not be removed without reducing the sample to negligible size. A large sample like CIG can be refined and quantified in terms of degree of isolation. It can then be correlated with multiwavelength interstellar medium (ISM) properties. The result can be a sample large enough to characterize the low density tail of the two-point correlation function. The result will also tell us: a) if truly isolated galaxies exist; b) in what numbers; and c) the environmental level where the onset of interaction-induced activity can first be detected. This study constitutes the AMIGA project (Analysis of the Interstellar Medium of Isolated Galaxies). AMIGA is compiling data that will characterise the all phases of the ISM and it is being released and periodically updated at http://www.iaa.csic.es/AMIGA.html.

This paper studies the optical properties of the entire CIG as the foundation sample for AMIGA. These properties can be compared with future refinements to monitor changes and possible biases in the statistical properties. The CIG is now almost redshift-complete allowing study of both apparent and distance-dependent properties. We first consider the distribution of CIG galaxies in 2 and 3 dimensions. This allows us to decide which local large scale-components contribute most heavily to the sample and how close the CIG comes to showing homogeneity. We then analyze sample completeness via the $V / V_{\mathrm{m}}$ test followed by derivation of the optical luminosity function (OLF). This is a much more reliable derivation than previous estimates because of the near completeness of redshift measures. Finally, we compare the OLF derived for the CIG sample with those of other samples representing different environments.

\section{The foundation of AMIGA: The original CIG catalog}

Statistical studies of isolated galaxies require a large, preselected and morphologically diverse sample. The tendency for galaxies to aggregate in multiple systems and clusters at all scales might suggest that such a sample is difficult to find. However Karachentseva (1973) compiled the Catalog of Isolated Galaxies (CIG) which includes 1051 objects. All of the CIG objects are found in the Catalog of Galaxies and Clusters of Galaxies (Zwicky et al. 1961-1968; CGCG) with $m_{\mathrm{pg}}<$ 15.7 and $\delta>-3^{\circ}, \sim 3 \%$ of the CGCG). Only one of the compiled objects is not a galaxy, but a globular cluster (CIG $781=$ Palomar 15, Nilson 1973), so the size of the sample considered in the rest of this paper is $n=1050$. We used the CIG as our starting point because it has a number of strengths:

- Size: the sample is large with $n=1050$ galaxies. This means that after refinement we will still be left with a statistically useful sample of several hundred galaxies.

- Isolation: the CIG sample was assembled with the requirement that no similar sized galaxies with diameter $d$ (between $1 / 4$ and 4 times diameter $D$ of the CIG galaxy) lie within $20 d$. Therefore for a CIG galaxy with $D=3^{\prime}$, no neighbor with $d=12^{\prime}$ may lie within $240^{\prime}$ and no companion with $d=0.75^{\prime}$ may lie within $15^{\prime}$. It is immediately seen that this criterion is superior to one with a fixed isolation in terms of the diameter of the CIG galaxy in question. However it is also clear that dwarf companions are not excluded. This is the familiar compromise between seeking isolation and avoiding the background of distant unrelated galaxies. There is no other way, in the absence of velocity data, to assemble a reasonably sized catalog of reasonably isolated galaxies. If one assumes an average $D=25 \mathrm{kpc}$ for a CIG galaxy and a typical "field" velocity $V=150 \mathrm{~km} \mathrm{~s}^{-1}$ then an approximately equal mass perturber would require $3 \times 10^{9}$ years to traverse a distance of $20 \mathrm{~d}$. While CIG likely contains many of the most isolated galaxies in the local Universe it is not biased for galaxies in voids because we are usually looking through the front side of the bubble of galaxies surrounding the void. Thus void galaxies often fail the isolation requirement.

- Complementarity: this CIG is complemented by catalogs of galaxy pairs (CPG, Catalog of Paired Galaxies; Karachentsev 1972), triplets (Karachentseva et al. 1979) and compact groups (Hickson catalog of Compact Groups, HCG; Hickson 1982; largely quartets). All of these interacting comparison samples were visually compiled using an isolation criterion. None of them take into account more hierarchical systems for the same reason that CIG could not do it. All avoid the pitfalls associated with computer compilation from a magnitude-limited catalog (i.e. selecting the brightest galaxy or galaxies in a cluster).

- Morphology: all morphological types are found in the CIG including a significant local supercluster dwarf population. The CIG sample is large enough to permit discrimination on the basis of galaxy type including approximately 100 non-dwarf early-type systems (see e.g. Aars et al. 2001; Marcum et al. 2004). It is also large enough to survive 
isolation re-evaluation that may reveal many additional interacting systems.

- Depth: the CIG samples a large enough volume of space to allow us to sample the majority of the optical luminosity function (OLF). Galaxies with a recession velocity less than $1000 \mathrm{~km} \mathrm{~s}^{-1}$ include the most isolated nearby dwarfs. Significant sampling at and beyond $10000 \mathrm{~km} \mathrm{~s}^{-1}$ allows us to also sample the extreme bright end of the OLF.

- Completeness: previous work suggested that the CIG is 80-90\% complete to $m_{\mathrm{Zw}} 15.0$ (XS91). See also Sect. 4.2.

\section{Refinements of the sample}

The CIG can be improved in several ways that take advantage of the digitized sky surveys (POSS1 and POSS2). Our two largest refinements include uniform reevaluation of morphology and isolation degree. This is being done for the entire sample except for the nearest dwarf galaxy subpopulation where numerous sources of distances and morphologies now exist.

\subsection{Morphology and positions}

The first papers discussing CIG morphology, and isolated galaxy morphology in general, are roughly contemporary with the appearance of the catalog in 1973. Galaxy classification data for CIG galaxies is non-uniform and often contradictory. According to the NED and LEDA databases the CIG is composed of $\sim 20 \%$ early types (E+S0), however the distribution of the individual mophologies shows a large discrepancy, as shown in Fig. 1. We re-evaluated CIG morphologies using the POSS2 images (Sulentic et al. in preparation) and find it possible to obtain reliable galaxy types for $80 \%$ or more of the sample. The population of luminous isolated spirals are the easiest to classify: near face-on spirals could be easily recognized beyond $10000 \mathrm{~km} \mathrm{~s}^{-1}$. The remaining $20 \%$ of the sample are being supplemented with archival data (e.g. SDSS; James et al. 2004) or new CCD images on 1-2 m class telescopes. POSS2 provides the higher spatial resolution necessary to distinguish between basic subtypes.

Comparing CIG positions in the SIMBAD database and the Updated Zwicky Catalogue (UZC; Falco et al. 2000) we found differences of up to several tens of arcsec for some galaxies, large enough to make accurate telescope pointings or cross correlations with on-line databases impossible. This motivated us to systematically revise all of the CIG positions using SExtractor on the images of the digitized sky surveys (Leon \& Verdes-Montenegro 2003). We found differences between old and new positions of up to $38.9^{\prime \prime}$ with a mean value of $2.4^{\prime \prime}$ for both SIMBAD and UZC.

\subsection{Redshift and distances}

The fraction of CIG with measured redshift has almost doubled in the past 15 years. Our archival and bibliographic search

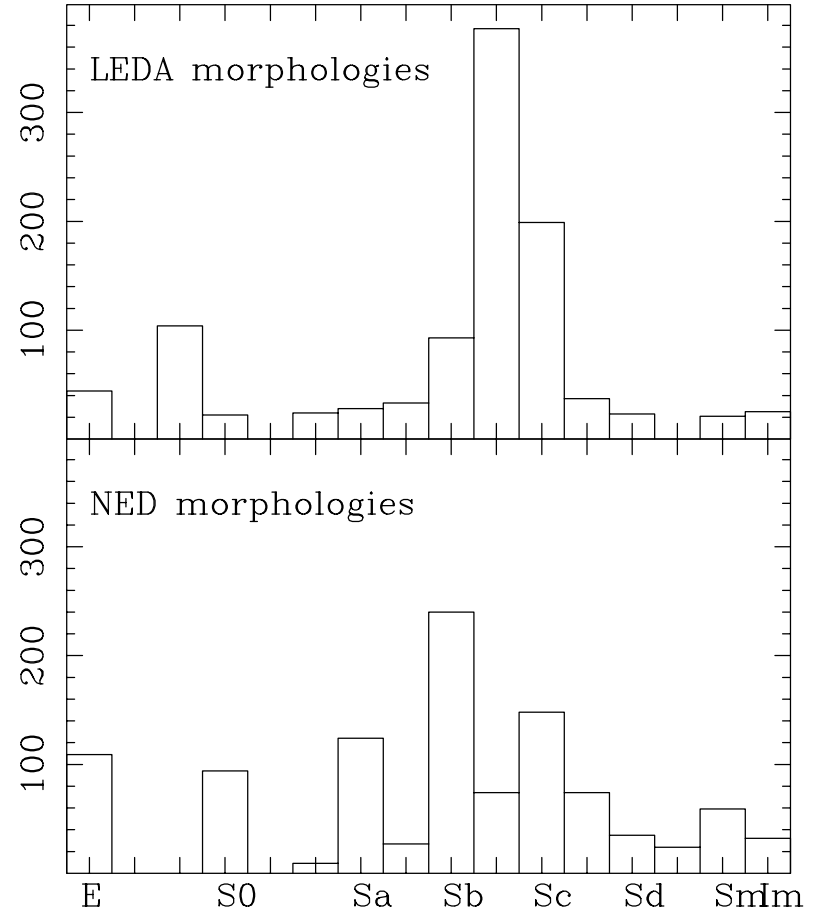

Fig. 1. Histogram of the morphological types of the full CIG sample obtained from NED (bottom) and LEDA (top).

reveals data for almost the entire sample (956/1050 galaxies $\left.^{1}\right)$. The redshift measures are given in Table 1. About one half of the redshifts were found in NED and we compiled the rest from 37 different sources. This includes 10 new HI observations from Nancay and Green Bank (see footnote to Table 1). Our search increased the CIG redshift sample by $n=489$ relative to the recent studies by Hernandez-Toledo et al. (1999, 2001). Redshift distances were derived for all galaxies with $V>1000 \mathrm{~km} \mathrm{~s}^{-1}$ and are expressed as $D=V_{3 \mathrm{~K}} / H_{0}$ where $V_{3 \mathrm{~K}}$ is the velocity after the $3 \mathrm{~K}$ correction (as given in Table 1 ) and assuming $H_{0}=75 \mathrm{~km} \mathrm{~s}^{-1} \mathrm{Mpc}^{-1}$. $3 \mathrm{~K}$ corrected velocities are computed in the reference frame defined by the $3 \mathrm{~K}$ cosmological background radiation. They are corrected for local velocity inhomogeneities due to the Local Group and Virgo Cluster. The velocity conversion is made with the standard correction as defined in Courteau \& Van den Bergh (1999). The velocity and apex directions of the Sun relative to the comoving frame have been derived from an analysis of the FIRAS data (Fixsen et al. 1996) with $V_{\text {apex }}=371 \mathrm{~km} \mathrm{~s}^{-1}$ and $\left(l_{\text {apex }}, b_{\text {apex }}\right)=$ $\left(264.14^{\circ}, 48.26^{\circ}\right)$. Redshift-independent distance estimates and references are provided for galaxies with $V<1000 \mathrm{~km} \mathrm{~s}^{-1}$ (Table 2), except for CIG $506\left(V=998 \mathrm{~km} \mathrm{~s}^{-1}\right), 657(V=$ $\left.626 \mathrm{~km} \mathrm{~s}^{-1}\right), 711\left(V=976 \mathrm{~km} \mathrm{~s}^{-1}\right), 748\left(V=961 \mathrm{~km} \mathrm{~s}^{-1}\right)$ and $753\left(V=851 \mathrm{~km} \mathrm{~s}^{-1}\right)$ listed in Table 1 since only redshiftderived distances could be obtained.

\footnotetext{
1 This number is updated in the electronic table at http://www.iaa.csic.es/AMIGA.html when new data become available.
} 
Table 1. Recession velocities for the CIG sample $\left(V_{\mathrm{r}}>1000 \mathrm{~km} \mathrm{~s}^{-1}\right)^{1}$.

\begin{tabular}{cllc}
\hline \hline CIG & $\begin{array}{l}V_{\mathrm{r}} \\
\mathrm{km} \mathrm{s}^{-1}\end{array}$ & $V_{3 \mathrm{~K}}$ & Reference $^{2}$ \\
\hline 1 & 7271 & 6914 & 1 \\
2 & 6983 & 6649 & 2 \\
4 & 2310 & 1959 & 3 \\
5 & 7865 & 7514 & 3 \\
6 & 4528 & 4183 & 3 \\
7 & 12752 & 12394 & 3 \\
. &.. &.. &.. \\
\hline
\end{tabular}

${ }^{1}$ Data are also given for the 5 CIG galaxies listed in Sect. 3.2 with $V_{\mathrm{r}}<1000 \mathrm{~km} \mathrm{~s}^{-1}$.

2 (1) Thereau et al. (1998), (2) Falco et al. (2000), (3) Huchra et al. (1999), (4) De Vaucouleurs et al. (1991; RC3), (5) SDSS early release 2001, (6) Giovanelli \& Haynes (1993), (7) Schneider et al. (1992), (8) Wegner et al. (1993), (9) Giovanelli et al. (1997), (10) Willick et al. (1990), (11) Kochanek et al. (2001), (12) Marzke et al. (1996), (13) Strauss et al. (1992), (14) Own data (HI spectra), (15) Fouque et al. (1992), (16) Karachentsev et al. (1981), (17) Huchra et al. (1990), (18) NED, (19) Beers et al. (1995), (20) Huchra et al. (1995), (21) Ugryumov et al. (2001), (22) Colless et al. (2001), (23) Schneider et al. (1990), (24) Mathewson \& Ford (1996), (25) Haynes et al. (1998), (26) Gavazzi et al. (1999), (27) Comte et al. (1999), (28) Van Driel (2000), (29) Haynes et al. (1997), (30) Grogin et al. (1998), (31) Kirshner et al. (1987), (32) Young (2000), (33) Saunders et al. (2000), (34) Freudling et al. (1992), (35) Haynes et al. (1999), (36) Fisher et al. (1995), (37) Lu et al. (1993), (38) Pietsch et al. (1998).

\section{Homogeneity, completeness and the optical luminosity function}

\subsection{Distribution on the sky and in velocity space}

Figure 2 shows the distribution of the CIG sample on the sky in $3000 \mathrm{~km} \mathrm{~s}^{-1}$ velocity intervals. This velocity segmentation makes it easier to recognize concentrations associated with major components of large-scale structure in the local Universe. The core of the Virgo cluster is indicated in the first segment with a circle of $D=12^{\circ}$. Other Abell clusters in the same redshift range and with richness classes 1 or 2 are indicated with circles corresponding to their core radius. As expected we see little correspondence between the positions of the nearby cluster cores and CIG galaxies. Of course some correspondence with more complex local large-scale structure components has been found (Haynes \& Giovanelli 1983). The 2-point correlation function for the CIG (Vettolani et al. 1986) also shows evidence for weak clustering.

Figure 3 shows the CIG redshift distribution which can be compared with earlier studies involving smaller parts of the sample (Haynes \& Giovanelli 1983; XS91) when far fewer redshifts were available. The earlier studies commented on evidence for large scale structure components including the local $\left(\sim 1500 \mathrm{~km} \mathrm{~s}^{-1}\right)$ and Pisces-Perseus $\left(\sim 5-6000 \mathrm{~km} \mathrm{~s}^{-1}\right)$ superclusters as well as the local void $\left(\sim 3000 \mathrm{~km} \mathrm{~s}^{-1}\right)$ surrounding the local supercluster. It is not clear that the latter void is
Table 2. Distances for the CIG sample $\left(V_{\mathrm{r}}<1000 \mathrm{~km} \mathrm{~s}^{-1}\right)$.

\begin{tabular}{rcc}
\hline \hline CIG & $\begin{array}{c}\text { Distance } \\
\text { Mpc }\end{array}$ & Reference $^{1}$ \\
\hline 45 & 4.8 & 1 \\
105 & 9.2 & 2 \\
109 & 10.3 & 3 \\
112 & 10.7 & 3 \\
121 & 7.8 & 4 \\
.. & $\ldots$ & $\ldots$ \\
\hline
\end{tabular}

1 (1) Karachentsev et al. (2003), (2) Terry et al. (2002), (3) Hamilton et al. (1996), (4) Parodi et al. (2002), (5) Miller et al. (2003), (6) Tully (1998), (7) Whiting (2003), (8) Freedman et al. (2001), (9) Sharina et al. (1999), (10) Bottema et al. (2002), (11) Garnett (2002), (12) Karachentsev et al. (1996). (13) Schmidt \& Boller (1992), (14) Karachentsev et al. (2003), (15) Gavazzi et al. (2000) (16) Sofue et al. (1998), (17) Teerikorpi et al. (1992), (18) Solanes et al. (2002), (19) Leonard et al. (2002), (20) Martin (1998), (21) Carrera et al. (2002), (22) Papaderos et al. (1996), (23) Swaters et al. (2002), (24) Bottinelli et al. (1986), (25) Bottinelli et al. (1985), (26) Majewski (1994), (27) Bellazzini et al. (2002), (28) Bottinelli et al. (1988), (29) Russell (2002), (30) Bottinelli et al. (1984).

actually seen. It is more appropriate to say that the level of the curve in the $3000 \mathrm{~km} \mathrm{~s}^{-1}$ region can be used to place an upper limit on any quasi-homogeneous component of the CIG. It is clear that the question of the existence of a galaxy "field" component is out of date. Large-scale structure dominates the distribution of galaxies and one can only try to isolate the lowest density regions of this structure. Figure 3 shows a comparison between the CIG redshift distribution and a corresponding homogeneous distribution of the same sample size with the same redshift distribution and Schechter luminosity function (see Sect. 4.3). It is clear that the fit is not satisfactory for $V<6000 \mathrm{~km} \mathrm{~s}^{-1}$ due to the above mentioned structure components. Removing these structures would provide an estimate of the fraction of CIG galaxies that is homogeneously distributed, at least in $2 \mathrm{D}$. In order to estimate this number we have assumed that the CIG is composed of both homogeneous and inhomogeneous populations. The latter is dominated by peaks at $1500 \mathrm{~km} \mathrm{~s}^{-1}$ and $5000 \mathrm{~km} \mathrm{~s}^{-1}$. We can fit a homogeneous distribution to the population underlying these peaks by scaling the solid curve downwards by a factor of 0.6 (dashed curve). Thus about half of the CIG sample can be argued to be reasonably homogeneous (see Figs. 2a,b). The solid curve fit to the complete sample approaches homogeneity at $\sim 6500 \mathrm{~km} \mathrm{~s}^{-1}$ corresponding to a volume of a radius of about $90 \mathrm{Mpc}$ ). The higher velocity part of the CIG samples a large enough volume to make sure that details of individual large-scale structure components have little effect on the velocity distribution. The residuals after subtraction of the underlying homogeneous (dashed curve) component show two peaks corresponding to the local and Pisces-Perseus Superclusters. Since the total CIG comprises about $3 \%$ of the CGCG this means that $1-2 \%$ of the CGCG can be argued to show homogeneity. This is about the same population fraction as the dense isolated compact 

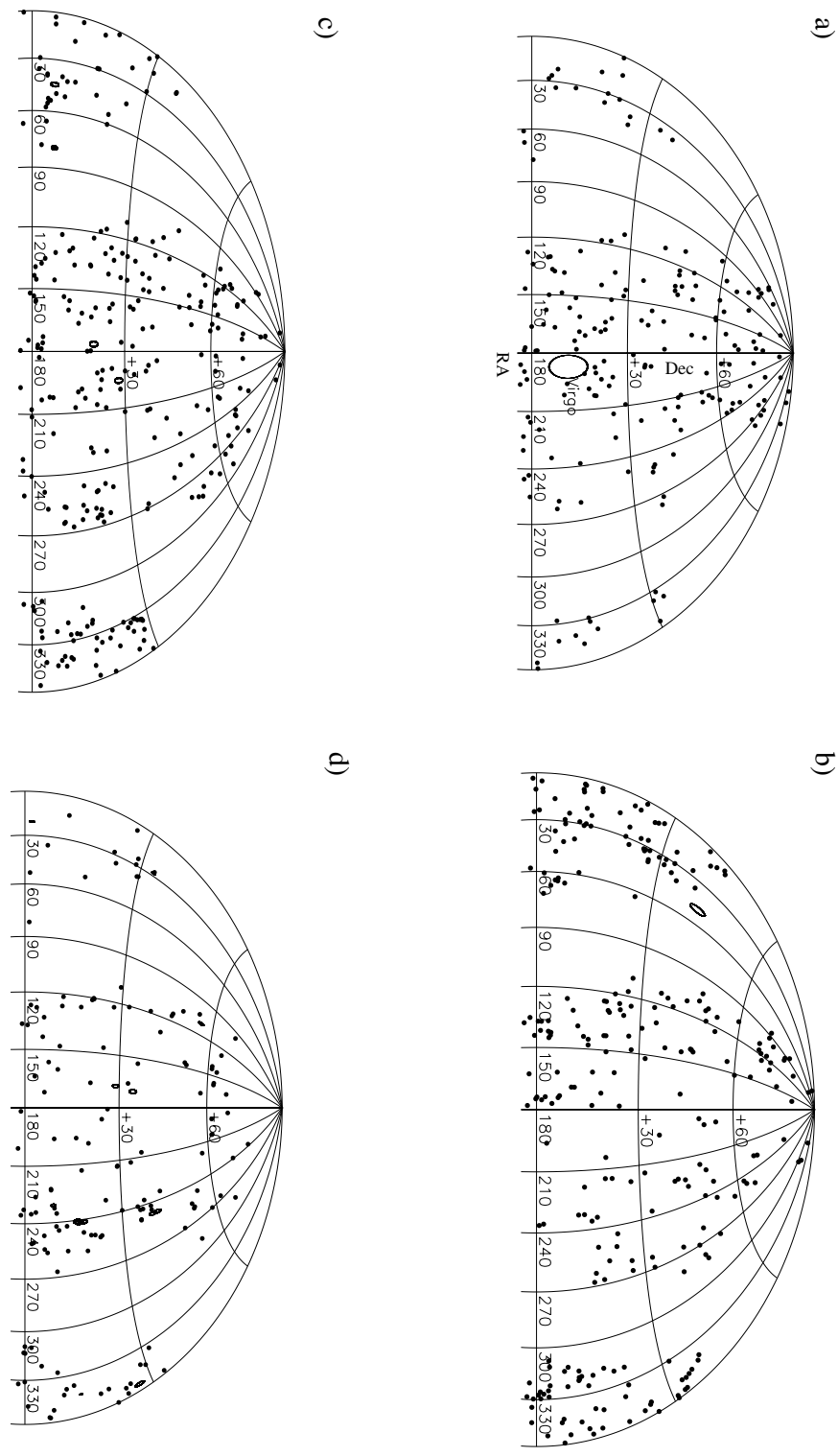

Fig. 2. Aitoff projection in right ascension and declination coordinates showing the distribution on the sky of CIG galaxies in the following velocity ranges. Galaxies in the $0-3000 \mathrm{~km} \mathrm{~s}^{-1}$ velocity interval are shown in a), 3000-6000 $\mathrm{km} \mathrm{s}^{-1}$ in b), 6000-9000 $\mathrm{km} \mathrm{s}^{-1}$ in c) and $9000-12000 \mathrm{~km} \mathrm{~s}^{-1}$ in d). The core of the Virgo cluster is indicated by a circle with $D=12^{\circ}$. Other Abell clusters in the same redshift range and with richness classes 1 or 2 are indicated by filled circles corresponding to their core radius.

groups (Mendes de Oliveira \& Hickson 1991, hereafter MH91; SR94) that lie at the other end of the "field" clustering spectrum. This fractional similarity is probably reasonable because both CIG (densest regions and least dense i.e. voids) and (e.g.) HCG share a similar avoidance of the most clustered regions via an isolation selection criterion (Sulentic 1987). The CIG is likely as close as we can hope to come to a local homogeneous component of the galaxy distribution.

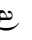

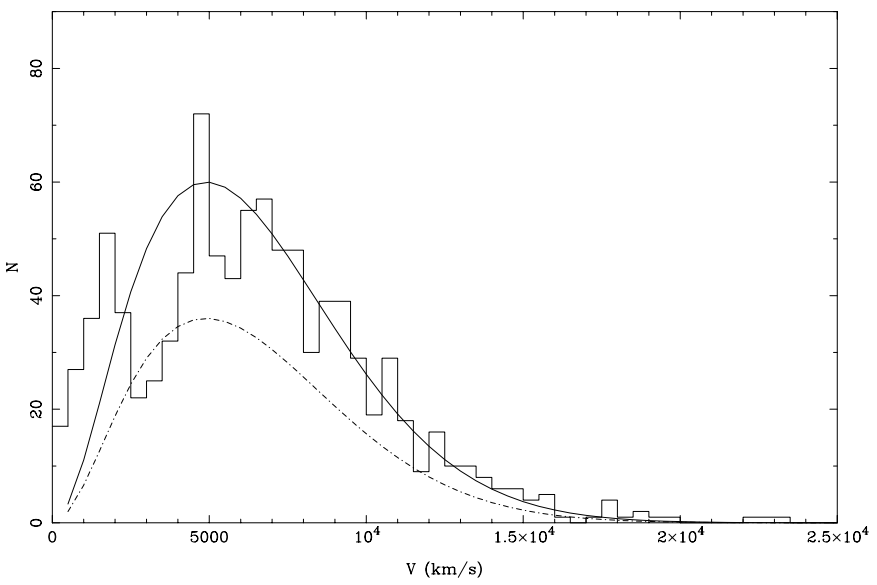

Fig. 3. Histogram of the optical heliocentric velocities of the 956 CIG galaxies with redshift data. Only CIG 402 is out of the plot, with $V=40658 \mathrm{~km} \mathrm{~s}^{-1}$. The solid line corresponds to a homogeneous redshift distribution of the same sample size, velocity distribution and Schechter function. The dashed line has been obtained by scaling down the previous distribution by a factor of 0.6.

\subsection{Optical magnitudes and completeness of the CIG sample}

We compiled blue magnitudes $\left(m_{B}\right)$ from the CGCG for all CIG galaxies and applied the following corrections.

- Systematic errors in the CGCG catalog were reported by Kron \& Shane (1976) (see also Giovanelli \& Haynes 1984), who showed that galaxies in Volume I of the CGCG had important systematic errors relative to the rest of volumes. We applied these corrections $\left(A_{\mathrm{v}}\right)$ to the CIG galaxies in Volume I (i.e. galaxies with $\delta<15$ degrees and $7 \mathrm{~h}<\alpha<$ $18 \mathrm{~h}$ ) and with $m_{B}$ up to $15.7 \mathrm{mag}$.

- Galactic dust extinction $\left(A_{\mathrm{g}}\right)$ has been derived from IRAS/DIRBE measurements of diffuse IR emission (Schlegel 1998).

- Internal extinction corrections $\left(A_{\mathrm{i}}\right)$ were calculated as a function of inclination and morphological type following $\mathrm{RC} 3$. Inclinations were estimated from the ratio of major to minor axes as given in NED. We used our revised morphologies (see Sect. 3.1).

- K corrections (Pence 1976; Giovanelli et al. 1981) were applied with a mean value of $0.05 \mathrm{mag}$, ranging from 0 to 0.3 mag depending on the morphological type.

In summary, the corrected $m_{B}$ was calculated as follows:

$m_{B-\text { corr }}=m_{B}+A_{\mathrm{v}}+A_{\mathrm{g}}+A_{\mathrm{i}}+A_{\mathrm{K}}$.

We list in Table 3 the CIG uncorrected and corrected magnitudes, as well as the optical luminosities, derived as

$\log \left(L_{B} / L_{\odot}\right)=12.192+2 \log [D(\mathrm{Mpc})]-0.4 m_{B-\text { corr }}$

We compared our corrected Zwicky magnitudes $m_{B \text {-corr }}$ with the $B_{\mathrm{T}}^{0}$ values for $507 \mathrm{CIG}$ galaxies found in RC3. The comparison is shown in Fig. 4. A regression analysis shows that both quantities are linearly related as:

$m_{B-\mathrm{corr}}=B_{\mathrm{T}}^{0}+0.136( \pm 0.001)$ 
Table 3. Optical magnitudes and luminosities of the CIG sample.

\begin{tabular}{cllr}
\hline \hline CIG & $\begin{array}{l}m_{B} \\
\mathrm{mag}\end{array}$ & $\begin{array}{l}m_{B-\mathrm{corr}} \\
\mathrm{mag}\end{array}$ & $\begin{array}{r}L_{B} \\
L_{\odot}\end{array}$ \\
\hline 1 & 14.30 & 13.64 & 10.67 \\
2 & 15.70 & 15.23 & 10.00 \\
3 & 15.70 & 15.04 & - \\
4 & 12.70 & 11.55 & 10.40 \\
5 & 15.50 & 14.52 & 10.39 \\
6 & 14.50 & 13.69 & 10.21 \\
7 & 15.60 & 15.30 & 10.51 \\
8 & 15.40 & 14.18 & 10.32 \\
9 & 15.40 & 14.54 & 10.45 \\
. &.. &.. &.. \\
\hline
\end{tabular}

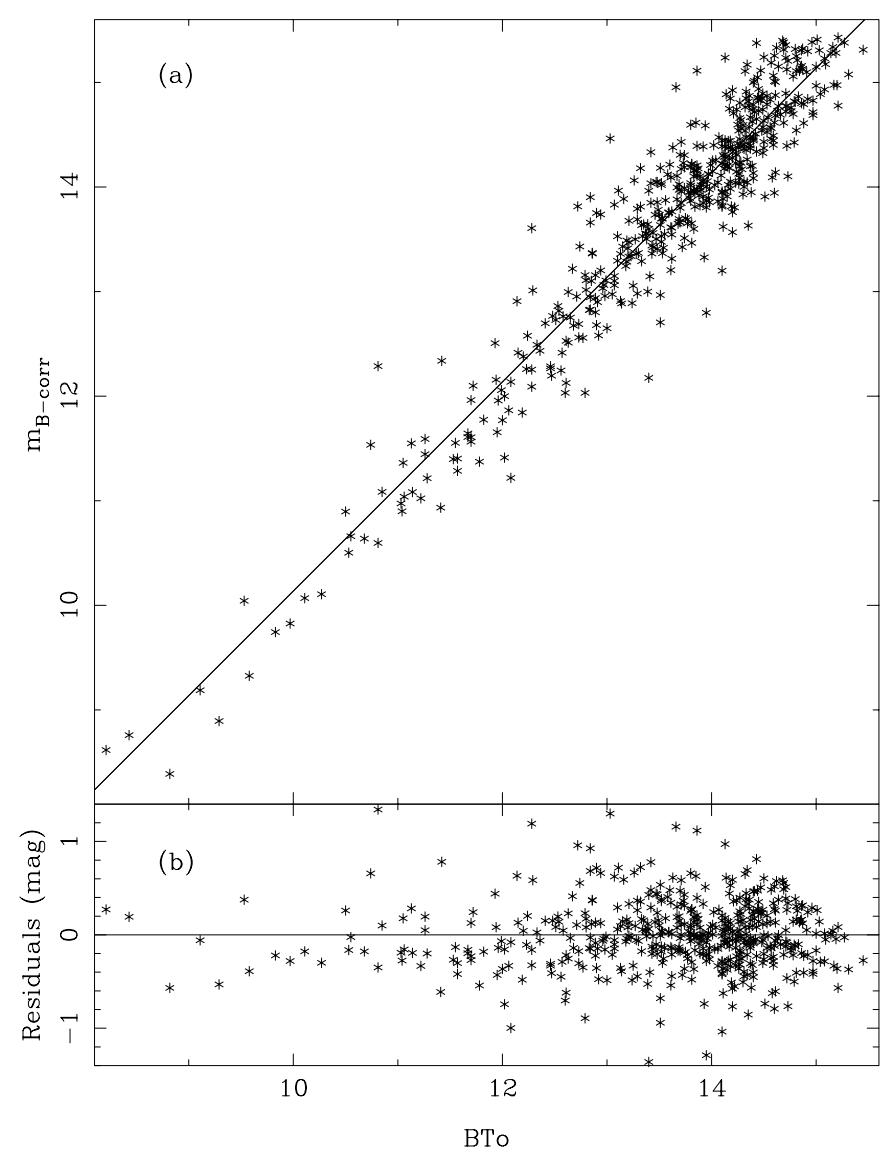

Fig. 4. a) Zwicky-corrected magnitudes versus $B_{\mathrm{T}}^{0}$ from $\mathrm{RC} 3$ for the 507 CIG galaxies in common. The solid line is a fit to the plotted data and the parameters of the fit are given in the text (Sect. 4.2). b) Residuals from the previous fit in magnitudes versus $B_{\mathrm{T}}^{0}$.

with a correlation coefficient of 0.96 . This gives confidence that our values are consistent with the RC3.

Figure 5 shows the distribution of apparent corrected magnitudes for the complete sample. We find only a few galaxies ( $n=19$ ) brighter than $m_{B \text {-corr }}=11.0$. This weak tail extending to $m_{\text {app }}=8.5$ represents galaxies that are in a sense interlopers to the CIG. They are the few large and bright galaxies in

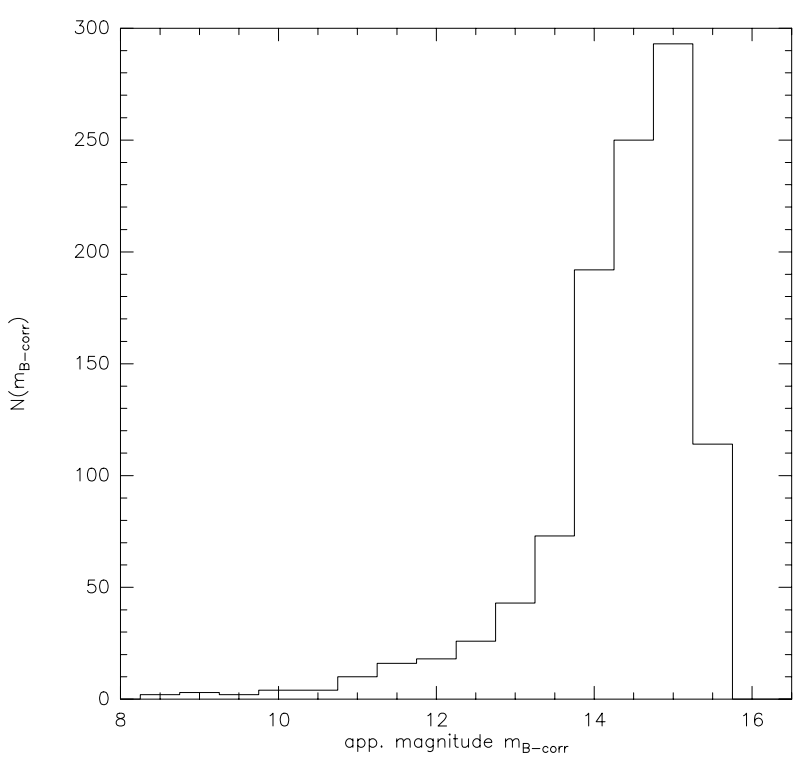

Fig. 5. Distribution of the corrected apparent magnitudes $(n=1050)$.

the nearby Universe that escaped deletion by the isolation criterion. They are almost certainly less isolated than the bulk of the CIG.

We used the $\left\langle V / V_{\mathrm{m}}\right\rangle$ test (Schmidt 1968) to evaluate CIG sample completeness. We calculate for each object the volume $V$ contained in a sphere whose radius is the distance to the object and the maximum volume $V_{\mathrm{m}}$ contained in a sphere whose radius is the maximum distance at which the galaxy would still be visible given the magnitude limit of the CIG. We then calculate the average of the objects brighter than the magnitude limit. This calculation is sensitive to the choice of the brightest magnitudes included. We choose to neglect galaxies brighter than 11 mag because they are obviously incomplete due to their small number per magnitude bin (see Fig. 4). This was also noted in the $\left\langle V / V_{\mathrm{m}}\right\rangle$ value when including them. It was not necessary to exclude galaxies fainter than 11 mag as they proved to be reasonably complete. Figure 6 shows the cumulative $\left\langle V / V_{\mathrm{m}}\right\rangle$ distribution as a function of limiting apparent magnitude. Results are presented for the complete sample $(n=1031)$ and the subsample for which recession velocities are available $(n=937)$, where galaxies brighter than $11 \mathrm{mag}$ have been excluded. The difference between the two samples is small and only visible at fainter magnitudes where the sample becomes slightly more complete. Our test suggests that the CIG is surprisingly complete (between 80-95\%) brighter than 15.0 . The sample becomes rapidly less complete at fainter magnitudes. We therefore adopted $m=15.0\left(\left\langle V / V_{\mathrm{m}}\right\rangle=0.41\right.$ for the sample with recession velocities) as the cutoff for inclusion in the sample used to derive the OLF. Hence the OLF has been calculated using $n=725$ galaxies which corresponds to 734 galaxies with known distance and magnitudes in the range 11-15 mag minus 9 galaxies with very high or low luminosity excluded since they were scattered in bins containing a low number of galaxies. In Table 4 we tabulate $\left\langle V / V_{\mathrm{m}}\right\rangle$ as a function of both apparent and absolute magnitudes for the redshiftcomplete sample. The high and low luminosity bins contain few sources because the former are rare and the latter are 
Table 4. $\left\langle V / V_{\mathrm{m}}\right\rangle$ for different limiting magnitudes and different absolute magnitude bins.

\begin{tabular}{c|cccc}
\hline \hline & \multicolumn{4}{|c}{ Limiting apparent magnitude } \\
\hline $\begin{array}{c}\text { Absolute magnitude } \\
M_{B-\text { corr }}\end{array}$ & 14.0 & 14.5 & 15.0 & 15.5 \\
\hline-23.0 & $0.550(2)$ & $0.276(2)$ & $0.138(2)$ & $0.069(2)$ \\
-22.0 & $0.580(15)$ & $0.514(28)$ & $0.477(51)$ & $0.291(57)$ \\
-21.0 & $0.536(82)$ & $0.500(160)$ & $0.426(253)$ & $0.275(296)$ \\
-20.0 & $0.461(79)$ & $0.480(155)$ & $0.423(248)$ & $0.315(319)$ \\
-19.0 & $0.403(46)$ & $0.394(74)$ & $0.344(102)$ & $0.329(146)$ \\
-18.0 & $0.458(25)$ & $0.382(38)$ & $0.255(44)$ & $0.232(54)$ \\
-17.0 & $0.540(7)$ & $0.379(9)$ & $0.465(18)$ & $0.354(25)$ \\
-16.0 & $0.385(5)$ & $0.389(8)$ & $0.374(11)$ & $0.456(22)$ \\
\hline All & $0.479(264)$ & $0.461(478)$ & $0.405(734)$ & $0.303(931)$ \\
\hline
\end{tabular}

The numbers in parenthesis give the number of galaxies in each bin ranging from $M_{B \text {-corr }}-0.5$ mag to $M_{B-\text { corr }}+0.5$ mag. All galaxies with velocity information and with apparent magnitudes between 11 and and the value indicated in the header of each column are included. The sum of the individual bin is somewhat smaller than the total number because the magnitudes of some galaxies fall outside the range considered.

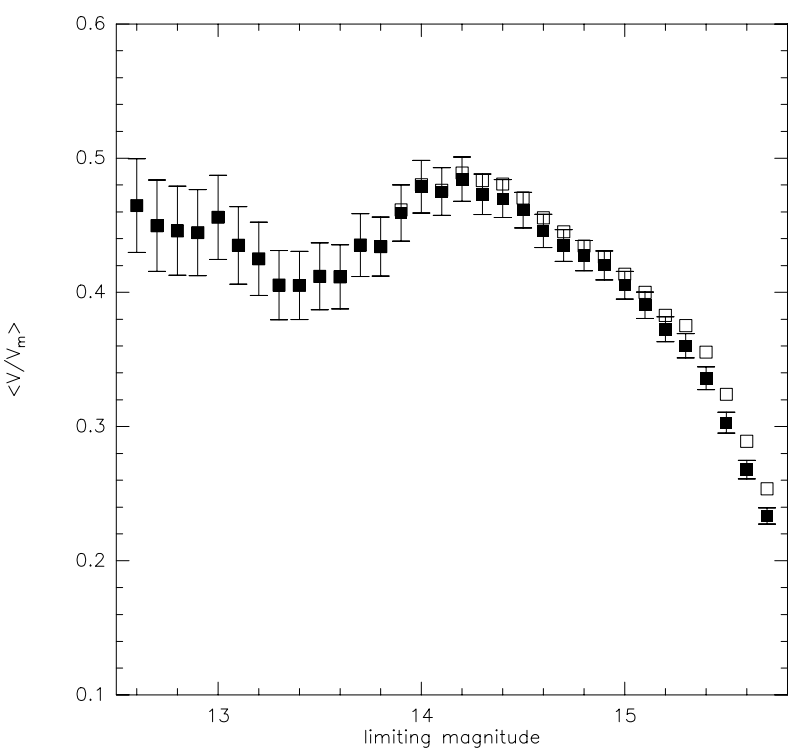

Fig. 6. The $\left\langle V / V_{\mathrm{m}}\right\rangle$ test for the CIG sample excluding galaxies brighter than 11 mag. The open squares indicate the whole sample $(n=1031)$, and the filled square the subsample for which recession velocities are available $(n=937)$ and which are used in the following to construct the OLF. The error bars are given for the latter subsamples and are the statistical errors in the mean $\left\langle V / V_{\mathrm{m}}\right\rangle$.

restricted to the extreme low redshift part of our sample. The depression centered at about limiting magnitude $\sim 13.3$ reflects the gap between our local supercluster and large-scale structure components beyond.

\subsection{CIG optical luminosity function}

The surprising level of completeness found for the CIG highlights its strength as mentioned earlier. The completeness correction is done by calculating $\left\langle V / V_{\mathrm{m}}\right\rangle$ in intervals of $0.1 \mathrm{mag}$ and adding the necessary number of galaxies in order to obtain for $\left\langle V / V_{\mathrm{m}}\right\rangle$ a value of $\sim 0.5$, characteristic of a complete homogeneous sample (see e.g. Huchra \& Sargent 1973). The galaxies added in each bin are taken into account when calculating $\left\langle V / V_{\mathrm{m}}\right\rangle$ for the next fainter bin. The final result depends somewhat on the bin size because a change in this parameter affects the assumed magnitude distribution of the added galaxies. Here we choose to make the smallest bin size commensurate with the precision of the data which corresponds roughly to the expected error in the adopted apparent magnitudes. Table 5 gives the results. We see that we have added 716 galaxies in order to make the sample complete to $m_{\text {app }}=15.0$. This yields a correction factor $\xi=$ of 2.0. We can estimate the uncertainty of this value due to the adopted bin size. If we had chosen a bin size of 0.2 or $0.05 \mathrm{mag}$ the number of added galaxies would have changed to 532 and 851 respectively. These correction factors would have been 1.7 and 2.2 respectively. We estimate an uncertainty of about $15 \%$.

The differential OLF $\Phi(M)$ estimating the number of galaxies per unit volume and per unit absolute magnitude level, is estimated from (Felten 1976)

$$
\Phi(M)=\frac{4 \pi}{\Omega} \frac{\xi}{\Delta M} \sum_{i} \frac{1}{V_{\mathrm{m}}\left(M_{i}\right)}
$$

where $\Omega$ is the sky coverage of the sample (4.38 sr for the CIG sample from XS91) and $V_{\mathrm{m}}\left(M_{i}\right)$ is the maximum volume within which a source of absolute magnitude $M_{i}$ could have been detected in a survey down to $m_{\mathrm{lim}}$, the limiting magnitude of the sample (here: $15 \mathrm{mag}$ ). The summation is over the luminosity interval $M_{i}+0.5 \triangle M \geq M \geq M_{i}-0.5 \triangle M$. We have chosen $\triangle M=0.5$. $\xi$ is the correction factor for incompleteness derived above. We give the so obtained OLF in Table 6. The variance of $\Phi(M)$ is estimated from

$\sigma^{2}=\left(\frac{4 \pi}{\Omega} \frac{\xi}{\Delta M}\right)^{2} \sum_{i} \frac{1}{V_{\mathrm{m}}^{2}\left(M_{i}\right)}$

Two previous estimates of the "field" OLF have been made using the CIG (both given in XS91 but partially revised in SR94). 
Table 5. Correction for incompleteness.

\begin{tabular}{|c|c|c|c|c|}
\hline Limiting mag. & $\left\langle V / V_{\mathrm{m}}\right\rangle$ & No. of gal. & Added gal. & Total add. gal. \\
\hline 12.100 & 0.546 & 34 & 0 & 0 \\
\hline 12.200 & 0.536 & 39 & 0 & 0 \\
\hline 12.300 & 0.532 & 45 & 0 & 0 \\
\hline 12.400 & 0.484 & 47 & 2 & 2 \\
\hline 12.500 & 0.460 & 51 & 3 & 5 \\
\hline 12.600 & 0.465 & 58 & 1 & 6 \\
\hline 12.700 & 0.450 & 63 & 3 & 9 \\
\hline 12.800 & 0.446 & 70 & 4 & 13 \\
\hline 12.900 & 0.444 & 78 & 3 & 16 \\
\hline 13.000 & 0.456 & 89 & 3 & 19 \\
\hline 13.100 & 0.435 & 96 & 8 & 27 \\
\hline 13.200 & 0.425 & 105 & 8 & 35 \\
\hline 13.300 & 0.405 & 112 & 12 & 47 \\
\hline 13.400 & 0.405 & 123 & 11 & 58 \\
\hline 13.500 & 0.412 & 137 & 11 & 69 \\
\hline 13.600 & 0.412 & 151 & 15 & 84 \\
\hline 13.700 & 0.435 & 174 & 10 & 94 \\
\hline 13.800 & 0.434 & 193 & 18 & 112 \\
\hline 13.900 & 0.459 & 226 & 11 & 123 \\
\hline 14.000 & 0.479 & 264 & 11 & 134 \\
\hline 14.100 & 0.475 & 298 & 22 & 156 \\
\hline 14.200 & 0.484 & 345 & 18 & 174 \\
\hline 14.300 & 0.473 & 384 & 34 & 208 \\
\hline 14.400 & 0.470 & 431 & 35 & 243 \\
\hline 14.500 & 0.461 & 478 & 46 & 289 \\
\hline 14.600 & 0.446 & 521 & 61 & 350 \\
\hline 14.700 & 0.435 & 570 & 70 & 420 \\
\hline 14.800 & 0.427 & 625 & 80 & 500 \\
\hline 14.900 & 0.420 & 683 & 95 & 595 \\
\hline 15.000 & 0.405 & 734 & 121 & 716 \\
\hline 15.100 & 0.390 & 785 & 143 & 859 \\
\hline 15.200 & 0.373 & 831 & 173 & 1032 \\
\hline 15.300 & 0.360 & 883 & 195 & 1227 \\
\hline 15.400 & 0.336 & 916 & 244 & 1471 \\
\hline 15.500 & 0.303 & 931 & 295 & 1766 \\
\hline 15.600 & 0.268 & 937 & 343 & 2109 \\
\hline 15.700 & 0.233 & 937 & 393 & 2502 \\
\hline 15.800 & 0.203 & 937 & 444 & 2946 \\
\hline 15.900 & 0.177 & 937 & 501 & 3447 \\
\hline 16.000 & 0.154 & 937 & 566 & 4013 \\
\hline
\end{tabular}

The first involved $n=295$ galaxies from the Arecibo sample (hereafter AIG; Haynes \& Giovanelli 1984) and the second involving virtually all late-type CIG galaxies with available redshift up to $1990(n=450)$. Both used uncorrected Zwicky magnitudes. The SR94 revision the XS91 OLF transformed the photographic magnitudes to the de Vaucouleurs $B_{\mathrm{T}}$-system with corrections for internal and external extinction. Our derivation has two advantages: a) a 2-3× larger and reasonably complete sample, as well as b) the ability to make more
Table 6. Optical luminosity function.

\begin{tabular}{ccc}
\hline \hline$M_{\mathrm{Zw}}$ & $\Phi\left(\mathrm{Mpc}^{-3} \mathrm{mag}^{-1}\right)$ & $n$ \\
\hline-16.25 & $3.69 \mathrm{E}-03 \pm 1.26 \mathrm{E}-03$ & 9 \\
-16.75 & $2.04 \mathrm{E}-03 \pm 6.22 \mathrm{E}-04$ & 11 \\
-17.25 & $8.20 \mathrm{E}-04 \pm 3.16 \mathrm{E}-04$ & 7 \\
-17.75 & $9.06 \mathrm{E}-04 \pm 2.33 \mathrm{E}-04$ & 16 \\
-18.25 & $7.50 \mathrm{E}-04 \pm 1.43 \mathrm{E}-04$ & 28 \\
-18.75 & $4.90 \mathrm{E}-04 \pm 8.46 \mathrm{E}-05$ & 35 \\
-19.25 & $4.64 \mathrm{E}-04 \pm 5.76 \mathrm{E}-05$ & 67 \\
-19.75 & $2.88 \mathrm{E}-04 \pm 3.19 \mathrm{E}-05$ & 85 \\
-20.25 & $2.88 \mathrm{E}-04 \pm 2.30 \mathrm{E}-05$ & 163 \\
-20.75 & $1.40 \mathrm{E}-04 \pm 1.15 \mathrm{E}-05$ & 154 \\
-21.25 & $4.65 \mathrm{E}-05 \pm 4.76 \mathrm{E}-06$ & 99 \\
-21.75 & $1.05 \mathrm{E}-05 \pm 1.61 \mathrm{E}-06$ & 44 \\
-22.25 & $8.84 \mathrm{E}-07 \pm 3.39 \mathrm{E}-07$ & 7 \\
\hline
\end{tabular}

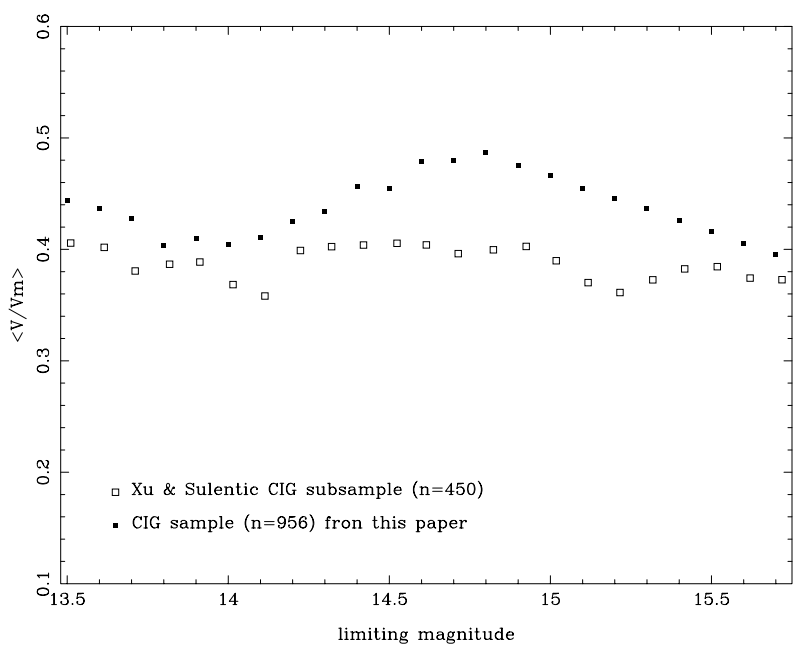

Fig. 7. The $\left\langle V / V_{\mathrm{m}}\right\rangle$ test for our CIG sample with available distances $(n=956)$ and for XS91 $(n=450)$ sample are shown respectively with filled and open squares. Our sample has been shifted by $0.5 \mathrm{mag}$ toward fainter values in order to match the uncorrected optical magnitudes used by XS91.

reliable magnitude corrections using revised morphologies. The average magnitude correction falls in the range 0.5-0.6 mag and shows up in a comparison of $\left\langle V / V_{\mathrm{m}}\right\rangle$ tests between our sample and previous analyses in XS91. In Fig. 7 we compare $\left\langle V / V_{\mathrm{m}}\right\rangle$ for our sample shifted by $0.5 \mathrm{mag}$ toward fainter values with $\left\langle V / V_{\mathrm{m}}\right\rangle$ from XS91. We find the largest difference in the range 14.5-15.5: this is the magnitude range where most of the $400+$ new redshifts obtained in the past $10+$ years are concentrated. $\left\langle V / V_{\mathrm{m}}\right\rangle$ appeared too flat above 15.0 in previous evaluations. Our derivation shows that many of these galaxies were actually brighter than 15.0. Our $\left\langle V / V_{\mathrm{m}}\right\rangle$ derivation (Fig. 6) shows a more natural decline towards fainter magnitudes.

We fit the OLF with a Schechter function:

$\Phi(M)=\Phi_{*} 10^{0.4(\alpha+1)\left(M^{*}-M\right)} \exp \left(-10^{0.4\left(M-M^{*}\right)}\right)$ 
Table 7. Optical luminosity function for the CIG sample.

\begin{tabular}{lcccc}
\hline \hline Sample & $\Phi\left(\mathrm{Mpc}^{-3} \mathrm{mag}^{-1}\right)$ & $\alpha$ & $M^{*}$ & Mag range for the fit \\
\hline CIG $n=725$ & $6.3( \pm 0.7) \times 10^{-4}$ & $-1.27 \pm 0.06$ & $-20.31 \pm 0.07$ & -16.3 to -22.3 \\
CIG $n=666$ & $7.5( \pm 0.6) \times 10^{-4}$ & $-0.82 \pm 0.09$ & $-20.11 \pm 0.07$ & -16.3 to -22.3 \\
$\left(V_{\mathrm{r}}<1500 \mathrm{~km} \mathrm{~s}^{-1}\right.$ excluded) & & & & \\
AIG (XS91) $n=280$ & $4.7 \times 10^{-4}$ & -1.4 & $-19.5^{a}$ & -16 to -21.8 \\
CIG (XS91) $n=450$ & $10.3 \times 10^{-4}$ & -1.4 & $-19.6^{a}$ & -16 to -21.8 \\
CIG (SR94) $n=450$ & $8.86( \pm 1.51) \times 10^{-4}$ & $-1.42 \pm 0.08$ & $-20.03 \pm 0.10^{b}$ & -15.25 to -21.8 \\
CIG (SR94) $M \leq-18.0$ & $1.11( \pm 0.20) \times 10^{-3}$ & $-1.20 \pm 0.08$ & $-19.89 \pm 0.12^{b}$ & -18 to -21.8 \\
\hline
\end{tabular}

${ }^{a}$ Uncorrected Zwicky magnitudes. The equivalence between these $M^{*}$ and our values for the CIG is explained in Sect. 4.3.

${ }^{b} \mathrm{~K}$ correction was not applied to these magnitudes.

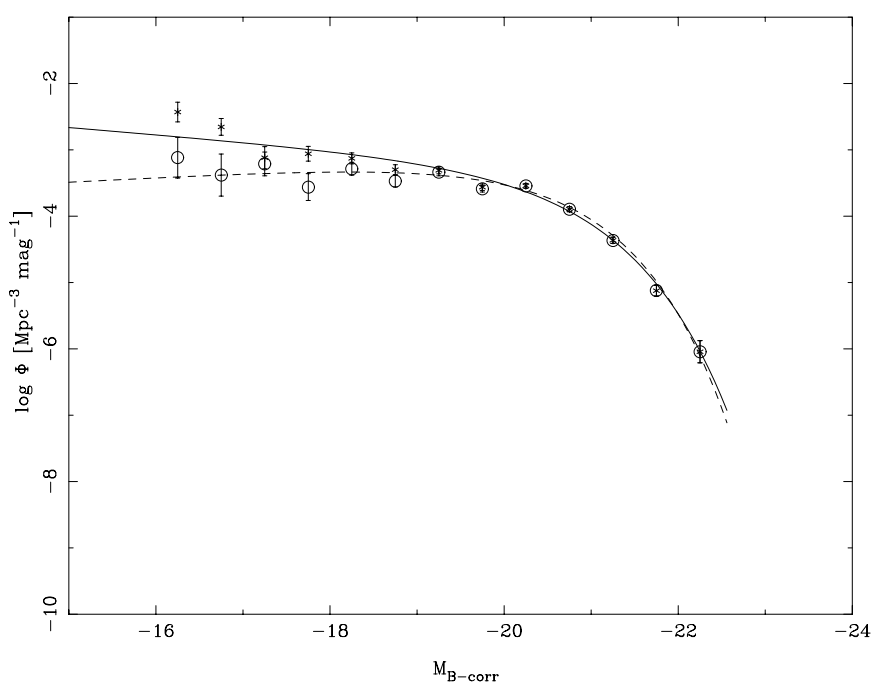

Fig. 8. Optical luminosity function for CIG galaxies for which velocity information exists and with apparent magnitudes between 11 and 15 $(n=725)$. The Schechter fit to this sample is plotted as a solid line. The dashed line corresponds to a fit to the same sample when galaxies with $V_{\mathrm{r}}<1500 \mathrm{~km} \mathrm{~s}^{-1}$ are removed.

using $n=725$ galaxies with known distance and magnitudes in the range 11-15, once having excluded 9 galaxies with very high or low luminosity scattered in bins containing a low number of galaxies. The fit is shown in Fig. 8 and the parameters are detailed in Table 7 where $M^{*}$ is given in corrected Zwicky magnitudes (see Sect. 3). The dip in our OLF at $M_{B}-18$ is likely related to the dips in the redshift distribution (Fig. 3) and $\left\langle V / V_{\mathrm{m}}\right\rangle$. All are related to the lack of homogeneity in our sample involving the local supercluster surrounded by a void and more distant structures. Our next step involved reproducing the two previous OLF determinations for the CIG indicated above. Figure 9 presents a comparison of all three OLF derivations involving $n=294$ (AIG), 450 (CIG, XS91) and 725 (CIG, our sample) galaxies respectively. We give all relevant fit parameters in Table 7 where $M^{*}$ for AIG and CIG (XS91) are given in uncorrected Zwicky magnitudes. We transformed $M^{*}$ from CIG (SR94) to Zwicky corrected magnitudes (SR94 magnitudes were not $\mathrm{K}$ corrected, a small effect of the order of $0.1 \mathrm{mag}$ ). The main difference oin our result (in agreement with SR94) with respect to $\mathrm{XS} 91$ is that our $M^{*}$ is $\sim 0.5 \mathrm{mag}$

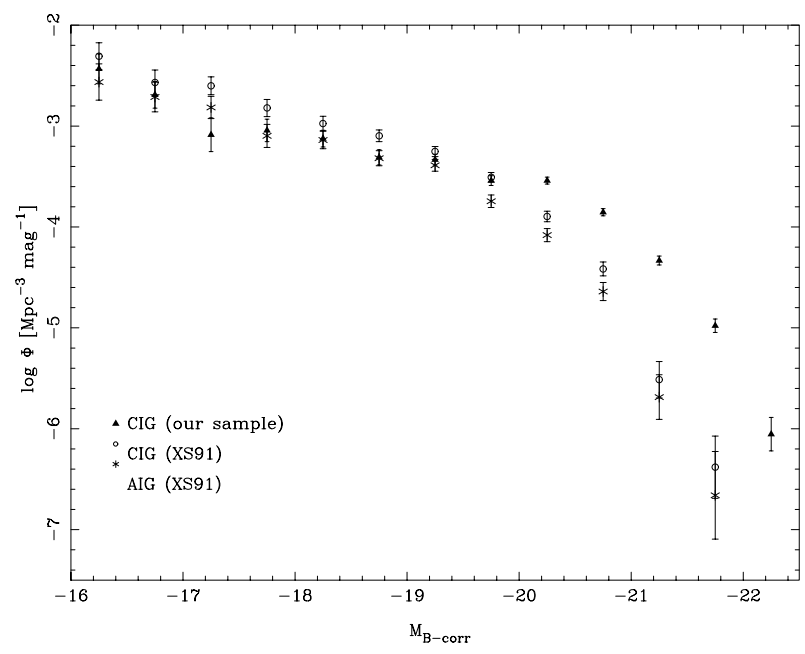

Fig. 9. All three OLF estimations for the CIG involving $n=725$ galaxies (our sample, filled triangles), 450 galaxies (CIG sample of XS91, open circles) and 294 galaxies (AIG sample of XS91, crosses). The CIG data are given in corrected magnitudes while the data of XS91 refer to uncorrected magnitudes.

brighter. This difference is primarily due to the magnitude corrections that we applied. Without this correction we were able to reproduce both of the previous OLF estimations within the error bars (Fig. 10). The small difference between our results and those of SR94 can be attributed to the absence of $\mathrm{K}$-correction in the latter.

The $\alpha$ parameter shows less difference to previous estimates. The underfit at the faint end results from the "interloper" population of dwarf galaxies mentioned earlier. All of the galaxies in the faintest two bins $(n=21)$ lie within a recession velocity $V_{\mathrm{r}}=2000 \mathrm{~km} \mathrm{~s}^{-1}$ and 13 of them within $V_{\mathrm{r}}=$ $1000 \mathrm{~km} \mathrm{~s}^{-1}$. Only a very small local volume is sensitive to such intrinsically faint galaxies. However many were found in this volume because of the ineffectiveness of the isolation criterion for galaxies within a few Mpc. We recalculated the OLF without local galaxies using several velocity cuts in addition to the restriction in magnitude range to $11-15$. When we remove galaxies with $V_{\mathrm{r}}<v_{\text {cut }} \mathrm{km} \mathrm{s}^{-1}$, where $v_{\text {cut }}$ ranges from 500 to $1500 \mathrm{~km} \mathrm{~s}^{-1}, \alpha$ changes systematically from -1.3 to -0.8 . Since all except 3 of the $n=59$ removed galaxies with $V_{\mathrm{r}}<1500 \mathrm{~km} \mathrm{~s}^{-1}$ are fainter than $M_{B-\text { corr }}=-20$ there is no 
Table 8. Optical luminosity function for the samples from the bibliography.

\begin{tabular}{|c|c|c|c|c|c|}
\hline Sample & Reference & $\Phi\left(\mathrm{Mpc}^{-3} \mathrm{mag}^{-1}\right)$ & $\alpha$ & $M^{*}$ & Mag range \\
\hline Field galaxies & Binggeli et al. & & -0.9 to -1.25 & -19.56 to -20.36 & $\leq-15.5$ to $\leq-18.5$ \\
\hline 2dFGRS all & Croton et al. (2004) & $8.95( \pm 0.05) \times 10^{-3}$ & $-1.05 \pm 0.02$ & $-20.33 \pm 0.02$ & -17.7 to -22.7 \\
\hline 2dFGRS void & $"$ & $1.32( \pm 0.56) \times 10^{-3}$ & $-1.06 \pm 0.24$ & $-19.52 \pm 0.16$ & \\
\hline 2dFGRS mean & $"$ & $9.62( \pm 1.0) \times 10^{-3}$ & $-0.99 \pm 0.04$ & $-20.12 \pm 0.05$ & \\
\hline 2dFGRS cluster & $”$ & $25.5( \pm 13.2) \times 10^{-3}$ & $-1.33 \pm 0.11$ & $-20.76 \pm 0.13$ & \\
\hline 2dFGRS cluster & De Propris et al. (2003) & & $-1.28 \pm 0.03$ & $-20.75 \pm 0.07$ & -15.7 to -23.2 \\
\hline SSRS2 & Marzke et al. (1998) & $5.4( \pm 2.0) \times 10^{-3}$ & $-1.12 \pm 0.05$ & $-20.33 \pm 0.06$ & -14.9 to -22.9 \\
\hline NOG All & Marinoni et al. (1999) & $5.9( \pm 0.9) \times 10^{-3}$ & $-1.10 \pm 0.06$ & $-20.53 \pm 0.08$ & -15.2 to -22.5 \\
\hline NOG Field & $"$ & & $-1.19 \pm 0.10$ & $-20.45 \pm 0.12$ & \\
\hline NOG Groups & $"$ & & $-1.02 \pm 0.07$ & $-20.63 \pm 0.10$ & \\
\hline NOG Groups $(n>10)$ & $"$ & & $-1.21 \pm 0.11$ & $-20.85 \pm 0.18$ & \\
\hline NOG Groups $(n>20)$ & $"$ & & $-1.28 \pm 0.18$ & $-20.86 \pm 0.31$ & \\
\hline CPG & SR94 & $2.60( \pm 0.24) \times 10^{-4}$ & $-0.90 \pm 0.09$ & $-20.34 \pm 0.06$ & \\
\hline $\mathrm{CPG} M \leq 18.0$ & SR94 & $2.31( \pm 0.22) \times 10^{-4}$ & $-1.06 \pm 0.07$ & $-20.24 \pm 0.07$ & \\
\hline HCGs & MH91 & $0.55_{-0.8}^{+0.2} \times 10^{-4}$ & $-0.2 \pm 0.9$ & $-20.11 \pm 0.20$ & \\
\hline HCGs & SR94 all & $1.82( \pm 0.33) \times 10^{-5}$ & $-1.13 \pm 0.12$ & $-20.09 \pm 0.11$ & \\
\hline HCGs & SR94 $M \leq 18.0$ & $9.21( \pm 2.72) \times 10^{-6}$ & $-1.69 \pm 0.13$ & $-20.53 \pm 0.15$ & \\
\hline HCGs & Zepf et al. (1997) & & $-0.80 \pm 0.15$ & $-19.99 \pm 0.16$ & -14.9 to -21.9 \\
\hline
\end{tabular}

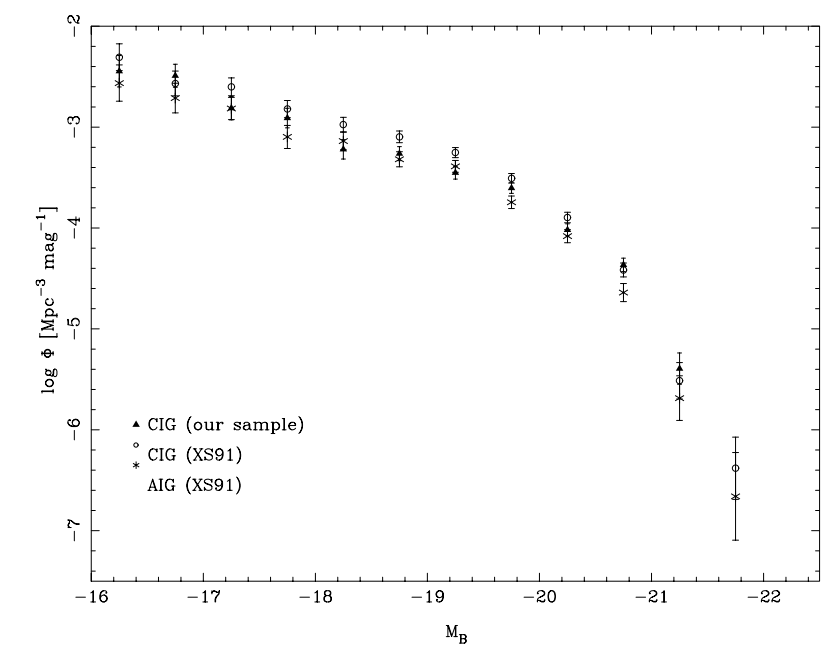

Fig. 10. The same as in Fig. 9 but calculated using uncorrected magnitudes for the CIG galaxies, as was done for the other two samples by XS91.

change in the OLF for magnitudes brighter than -20. However the fit to the bright part of the OLF changes because we are truncating our reasonably complete sample, resulting in a failure of the Schechter function to model the OLF properly. The only interesting result to emerge from this truncation involves the decrease in the $\alpha$ parameter. The fit obtained for $v_{\text {cut }}=1500$ is shown with a dashed line in Fig. 8 and the fit parameters are given in Table 7 .

\subsection{Comparison of the optical luminosity function of the CIG with other samples in the bibliography}

In this section we compare our CIG OLF with results of other samples involving a range of environments (Table 8). We have also included the results from the compilation of Binggeli et al. (1988) for the OLF of field galaxies. We selected several samples for comparison and concentrate on the shape of the OLF rather than the actual space density $(\Phi)$ which is an extremely difficult thing to compare. The absolute magnitude range covered by each sample is given in Table 8 . All published values of $M^{*}$ have been reduced to $H_{0}=75 \mathrm{~km} \mathrm{~s}^{-1} \mathrm{Mpc}^{-1}$ and transformed to Zwicky corrected magnitudes ( $\left.m_{B \text {-corr }}\right)$ using the appropriate relation: $m_{B-\text { corr }}=B_{\mathrm{MGC}}-0.124, m_{B-\text { corr }}=$ $g_{\mathrm{SDSS}}^{*}+0.276, m_{B-\mathrm{corr}}=b_{2 \mathrm{dFGRS}}-0.054, m_{B-\text { corr }}=b_{\mathrm{SSRS} 2}-$ 0.284 , obtained from the relation given in Eq. (3) combined with the transformations from Liske et al. (2003).

The comparison samples include:

- The Nearby Optical Galaxy (NOG) sample (Marinoni et al. 1999; $v<5500 \mathrm{~km} \mathrm{~s}^{-1}$ ). They distinguish subsamples according to various group properties (Garcia 1993) for a total of 4025 galaxies. Any galaxy not included in one of the group categories is considered "field". Hence their field sample likely contains interacting pairs. The morphologies were compiled by Garcia et al. (1993) from RC3. Adopted magnitudes were in the RC3 $B_{\mathrm{T}}$ system.

- The Second Southern Sky Redshift Survey (Marzke et al. 1998) samples a larger volume $(z<0.05)$ and contains $n=$ 5404 galaxies. This is a magnitude-limited sample without consideration of isolation degree. Morphological classifications come from several sources, ranging from detailed to rough designations. The $\left(b_{\mathrm{SSRS} 2}\right)$ magnitude system is calibrated with CCD photometry and defined to match the $B(0)$ system used in the CfA survey.

- The 2dFGRS survey samples an even larger volume (Croton et al. 2004; $z<0.11$ ) and includes $n=$ 81387 galaxies. They cover a wide range of environmental densities, defined as the density contrast in spheres of radius $R=8 \mathrm{Mpc}$. Morphologies were divided into late and 


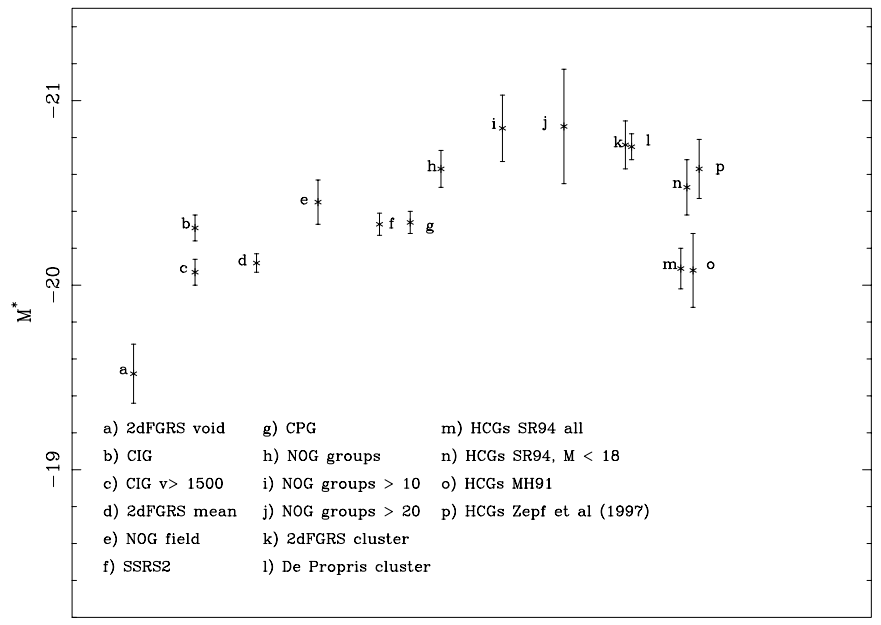

Approximate environment density $--->$

Fig. 11. The Schechter function $M^{*}$ parameter as a function of the environment, ordered in an approximate way. The label "all" indicates that the $M^{*}$ value has been obtained for all galaxies in the sample independently of the environment.

early types based on spectral type. Their photometric system $b_{\mathrm{J}}$ (noted here as $b_{2 \mathrm{dFGRS}}$ ) is based on the response of the Kodak IIIa-J +GG395 emulsion/filter combination with the zero point determined from Johnson $B$-band CCD photometry. De Propris et al. (2003) determined the OLF for galaxies in 60 clusters from the $2 \mathrm{dFGRS}$.

- The CPG and HCG are samples where close encounters are likely to dominate over effects of local galaxian density (i.e. they involve isolated pairs and compact groups). Both samples were selected using criteria similar to the ones employed in compiling the CIG. The CPG was used as a control sample in previous determinations of the CPG OLF (XS91; Toledo et al. 1999; Hernandez-Toledo et al. 1999) and contains 528 pairs. Original Zwicky magnitudes were converted to the $B_{\mathrm{T}}^{\mathrm{C}}$ system. The HCG OLF has been estimated by different authors. MH91 and SR94 considered a sample composed of 68 HCGs while Zepf et al. (1997) considered 17 HCGs together with galaxies in the close environment of the groups. MH91 and SR94 find: 1) a deficit of low luminosity galaxies (depressed $\alpha$ disputed by Zepf et al.); and 2) an excess of bright ellipticals and 3) near CIG-like behavior for the spiral population. The latter result is in strong contrast to the CPG OLF (XS91) where a significantly brighter $M^{*}$ was found and interpreted as the signature of interaction-induced star formation.

Figure 11 plots $M^{*}$ for each sample ordered roughly by environmental density. The sequence indicates reasonably clearly the change in OLF parameters as one proceeds from higher to lower density samples. The former show an excess of high luminosity galaxies as inferred from $M^{*}$. Our CIG value for $M^{*}$ betterfits somewhat denser environment than voids but with a lower density than some field estimates. This is consistent with the fact that the isolation criterion mitigates any possible bias towards inclusion of void members in the CIG. Void galaxies are often not isolated in projection but only in 3D. Even void

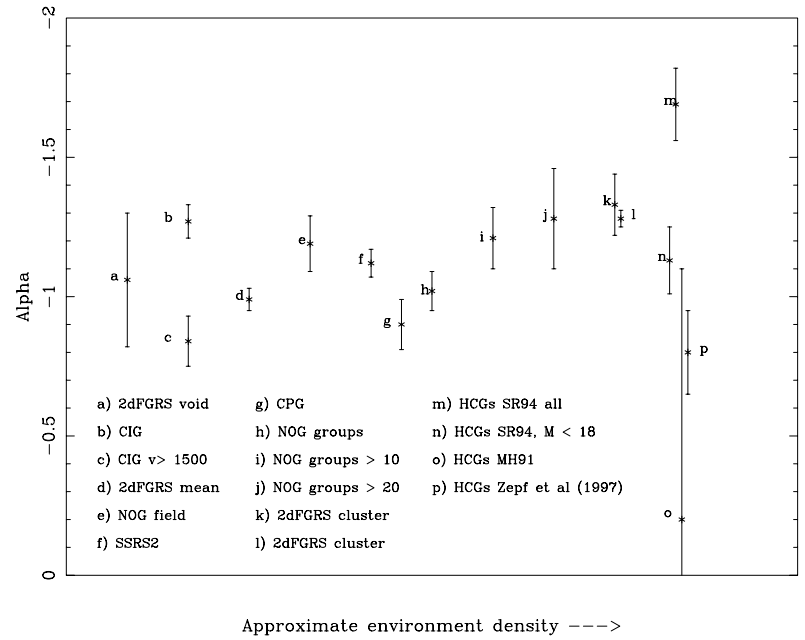

Fig. 12. The same as in Fig. 11 for the $\alpha$ parameter.

samples contain interacting pairs (e.g. Grogin \& Geller 2000) and $M^{*}$ will be affected depending on their fractional representation in the sample.

We also find a possible environmental trend (albeit with larger scatter) for the $\alpha$ parameter (Fig. 12) in the sense that it becomes more negative for denser environments. The location of the CIG in this plot obviously depends on the inclusion or exclusion of the local part of the sample $\left(V_{\mathrm{r}}<1500 \mathrm{~km} \mathrm{~s}^{-1}\right)$ which is dominated by low luminosity dwarf galaxies $(M>-19)$. Our results are consistent with Marinoni et al. (1999) when local dwarfs are included in the CIG which is reasonable since they sample galaxies with absolute magnitudes down to $M_{B}=$ -15.2. Results for the HCG are controversial since a significant dwarf population is only found if one increases the diameter of the groups as defined for the high luminosity members. While low luminosity CPG pairs are found, very few compact groups composed entirely of low luminosity members are found, for example, in the HCG (see SR94 Fig. 1).

\section{Concluding remarks}

The CIG sample is the basis of the AMIGA project. It has many advantages as a source of galaxies in low density environments, not the least of which is its relatively large size. This means that it can be refined without reducing the final sample population below a size that would be statistically useful. We find that its 2D distribution is reasonably homogeneous as we would expect for a distribution sampling, predominantly, the peripheries of large-scale structure features. It is affected by the local and Pisces-Perseus superclusters in 3D. The former because we are inside it and the latter because it is rather large and diffuse. Underlying these two bumps in the redshift distribution we again find evidence that $50 \%$ or more of the sample shows a quasi-homogeneous redshift distribution, motivating us to suggest that CIG is as close as we can hope to come towards achieving a local "field" population. A $V / V_{\mathrm{m}}$ test confirms the completeness of the CIG and a comparison of the OLF of the CIG with that of other samples re-enforces the credibility of the idea that CIG OLF is representative of the lower density parts of the galaxy environment. Care must be taken 
with the local supercluster contribution to the CIG because it samples the OLF to much lower luminosities than the rest of the sample.

Acknowledgements. L.V.-M., U.L., D.E., S.L., S.V. and E.G. are partially supported by DGI (Spain) AYA 2002-03338 and Junta de Andalucía TIC-114 (Spain).

\section{References}

Aars, C. E., Marcum, P. M., \& Fanelli, M. N. 2001, AJ, 122, 2923

Aceves, H., \& Velazquez, H. 2002, RMxAA, 38, 199

Adams, M. T., Jensen, E. B., \& Stocke, J. T. 1980, AJ, 85, 1010

Beers, T. C., Kriessler, J. R., Bird, C. M., \& Huchra, J. 1995, AJ, 109, 874

Bellazzini, M., Ferraro, F. R., Origlia, L., et al. 2002, AJ, 124, 3222

Boselli, A., Gavazzi, G., Donas, J., \& Scodeggio, M. 2001, AJ, 121, 753

Bottema, R., Pestaña, J. L. G., Rothberg, B., \& Sanders, R. H. 2002, A\&A, 393, 453

Bottinelli, L., Gouguenheim, L., Paturel, G., \& de Vaucouleurs, G. 1984, A\&AS, 56, 381

Bottinelli, L., Gouguenheim, L., Paturel, G., \& de Vaucouleurs, G. 1985, A\&AS, 59, 43

Bottinelli, L., Gouguenheim, L., Paturel, G., \& Teerikorpi, P. 1986, A\&A, 156, 157

Bottinelli, L., Gouguenheim, L., \& Teerikorpi, P. 1988, A\&A, 196, 17 Bushouse, H. A. 1987, ApJ, 320, 49

Carrera, R., Aparicio, A., Martinez-Delgado, D., \& Alonso-Garcia, J. 2002, AJ, 123, 3199

Colbert, J. W., Mulchaey, J. S., \& Zabludoff, A. I. 2001, AJ, 121, 808

Colless, M., Dalton, G., Maddox, S., et al. 2001, MNRAS, 328, 1039

Comte, G., Petrosian, A. R., Ohanian, G. A., \& Stepanian, J. A. 1999, Afz, 42,149

Courteau, S., \& van den Bergh, S. 1999, AJ, 118, 337

Croton et al. 2004 [arXiv:astro-ph 0407537]

Cruzen, S., Wehr, T., Weistrop, D., Angione, R. J., \& Hoopes, C. 2002, AJ, 123, 142

De Propris, R., Colless, M., Driver, S. P., et al. 2003, MNRAS, 342, 725

Drozdovsky, I. O., \& Karachentsev, I. D. 2000, A\&AS, 142, 425

Dultzin-Hacyan, D., Krongold, Y., Fuentes-Guridi, I., \& Marziani, P. 1999, ApJ, 513, L111

Falco, E. E., et al. 2000, The Updated Zwicky Catalog (UZC)

Felten, J. E. 1976, ApJ, 207, 700

Fisher, K. B., Huchra, J. P., Strauss, M. A., et al. 1995, ApJS, 100, 69

Fixsen, D. J., Cheng, E. S., Gales, J. M., et al. 1996, ApJ, 473, 576

Fouque, P. Durand, N., Bottinelli, L., Gouguenheim, L., \& Paturel, G. 1992, Catalogue of Optical Radial Velocities, Observatoires de Lyon et Paris-Meudon, 1, 1

Freedman, W. L., Madore, B. F., Gibson, B. K., et al. 2001, ApJ, 553, 47

Freudling, W., Haynes, M. P., \& Giovanelli, R. 1992, ApJS, 79, 157

Fuentes-Williams, T., \& Stocke, J. T. 1988, AJ, 96, 1235

Garcia, A. M. 1993, A\&AS, 100, 47

Garcia, A. M., Paturel, G., Bottinelli, L., \& Gouguenheim, L. 1993, A\&AS, 98, 7

Garnett, D. 2002, ApJ, 581, 1019

Gavazzi, G., Carrasco, L., \& Galli, R. 1999, A\&AS, 136, 227

Gavazzi, G., Franzetti, P., Scodeggio, M., Boselli, A., \& Pierini, D. 2000, A\&A, 361, 863

Giovanelli, R., \& Haynes, M. 1984, AJ, 89, 1

Giovanelli, R., \& Haynes, M. P. 1993, AJ, 105, 1271
Giovanelli, R., Haynes, M. P., \& Chincarini, G. L. 1981, ApJ, 247, 383

Giovanelli, R., Avera, E., \& Karachentsev, I. D. 1997, AJ, 114, 122

Gott, J. R., III, \& Turner, E. L. 1976, ApJ, 209, 1

Grogin, N. A., Geller, M. J., \& Huchra, J. P. 1998, ApJS, 119, 277

Grogin, N. A., \& Geller, M. J. 2000, AJ, 119, 32

Hamilton, T. T., Gotthelf, E. V., \& Helfand, D. J. 1996, ApJ, 466, 795

Haynes, M. P., \& Giovanelli, R. 1980, ApJ, 240, L87

Haynes, M. P., \& Giovanelli, R. 1983, ApJ, 275, 472

Haynes, M. P., \& Giovanelli, R. 1984, AJ, 89, 758

Haynes, M. P., Giovanelli, R., Herter, T., et al. 1997, AJ, 113, 1197

Haynes, M. P., van Zee, L., Hogg, D. E., Roberts, M. S., \& Maddalena, R. J. 1998, AJ, 115, 62

Haynes, M. P., Giovanelli, R., Chamaraux, P., et al. 1999, AJ, 117, 2039

Hernandez-Toledo, H. M., Dultzin-Hacyan, D., Gonzalez, J. J., \& Sulentic, J. W. 1999, AJ, 118, 108

Hernandez Toledo, H. M., Dultzin-Hacyan, D., \& Sulentic, J. W. 2001, AJ, 121, 1319

Hickson, P. 1982, ApJ, 255, 382

Huchra, J., \& Sargent, W. L. W. 1973, ApJ, 186, 433

Huchra, J., \& Thuan, T. X. 1977, ApJ, 216, 694

Huchra, J. P., Geller, M. J., de Lapparent, V., \& Corwin, H. G., Jr. 1990, ApJS, 72, 433

Huchra, J. P., Geller, M. J., \& Corwin, H. G., Jr. 1995, ApJS, 99, 391

Huchra, J. P., Vogeley, M. S., \& Geller, M. J. 1999, ApJS, 121, 287

James, P. A., Shane, N. S., Beckman, J. E., et al. 2004, A\&A, 414, 23

Joseph, R. D., \& Wright, G. S. 1985, MNRAS, 214, 87

Karachentsev, I. D. 1972, SoSAO, 7, 1

Karachentsev, I. D. 1987, Double Galaxies (Moscow: Nauka)

Karachentsev, I. D., Karachentseva, V. E., \& Mineva, V. A. 1981, SvAL, 7, 298

Karachentsev I. D., Musella, I., \& Grimaldi, A. 1996, A\&A, 310, 722

Karachentsev, I. D., Makarov, D. I., Sharina, M. E., et al. 2003, A\&A, 398, 479

Karachentseva, V. E. 1973, Comm. Spec. Ap. Obs., USSR 8, 1

Karachentseva, V. E., Karachentsev, I. D., \& Shcherbanovskii, A. L. 1979, AISAO, 11, 3

Karachentseva, V. E., Lebedev, V. S., \& Shcherbanovskij, A. L. 1986, Catalogoe of Isolated Galaxies. Bull. Inf. CDS, 30, 125

Kennicutt, R. C., \& Kent, S. M. 1983, AJ, 88, 1094

Kirshner, R. P., Oemler, A., Jr., Schechter, P., \& Shectman, S. A. 1987, ApJ, 314, 493

Kochanek, C. S., Pahre, M. A., Falco, E. E., et al. 2001, ApJ, 560, 566

Kron, G. E., \& Shane, C. D. 1976, Ap\&SS, 39, 401

Krongold, Y., Dultzin-Hacyan, D., \& Marziani, P. 2003, RMxAC, 17, 105

Larson, R. B., \& Tinsley, B. M. 1978, ApJ, 219, 46

Laurikainen, E., Salo, H., Teerikorpi, P., \& Petrov, G. 1994, A\&AS, 108, 491

Leon, S., \& Verdes-Montenegro, L. 2003, A\&A, 411, 391

Leonard, D. C., Filippenko, A. V., Gates, E. L., et al. 2002, PASP, 114, 35

Lequeux, J. 1971, A\&A, 15, 42

Liske, J., Lemon, D. J., Driver, S. P., Cross, N. J. G., \& Couch, W. J. 2003, MNRAS, 344, 307

Lu, N. Y., Hoffman, G. L., Groff, T., Roos, T., \& Lamphier, C. 1993, ApJS, 88, 383

Majewski, S. R. 1994, ApJ, 431, L17

Makarova, L. N., \& Karachentsev, I. D. 1998, A\&AS, 133, 181

Marcum, P. M., Aars, C. E., \& Fanelli, M. N. 2004, AJ, 127, 3213

Marinoni, C., Monaco, P., Giuricin, G., \& Costantini, B. 1999, ApJ, 521,50 
Marquez, I., \& Moles, M. 1999, A\&A, 344, 421

Marquez, I., Durret, F., Masegosa, J., et al. 2000, A\&A, 360, 431

Martin, M. C. 1998, A\&AS, 131, 73

Marzke, R. O., Huchra, J. P., \& Geller, M. J. 1996, AJ, 112, 1803

Marzke, R. O., da Costa, L. N., Pellegrini, P. S., Willmer, C. N. A., \& Geller, M. J. 1998, ApJ, 503, 617

Mathewson, D. S., \& Ford, V. L. 1996, ApJS, 107, 97

Mendes de Oliveira, C., \& Hickson, P. 1991, ApJ, 380, 30

Miller, S. T., \& Veilleux, S. 2003, ApJS, 148, 383

Moore, B., Katz, N., Lake, G., Dressler, A., \& Oemler, A., Jr. 1996, Nature, 379, 613

Morgan, I., Smith, R. M., \& Phillipps, S. 1998, MNRAS, 295, 99

Nilson, P. 1973, Uppsala General Catalogue of Galaxies, Acta Universitatis Upsalienis, ova Regiae Societatis Upsaliensis

Odewahn, S. C. 1995, PASP, 107, 770

Odewahn, S. C., Windhorst, R. A., Driver, S. P., \& Keel, W. C. 1996, ApJ, 472, 13

Papaderos, P., Loose, H.-H., Thuan, T. X., \& Fricke, K. J. 1996, A\&AS, 120, 207

Parodi, B. R., Barazza, F. D., \& Binggeli, B. 2002, A\&A, 388, 29

Pence, W. 1976, ApJ, 203, 39

Perea, J., del Olmo, A., Verdes-Montenegro, L., \& Yun, M. S. 1997, ApJ, 490, 166

Pietsch, W., Bischoff, K., Boller, T., et al. 1998, A\&A, 333, 48

Pisano, D. J., Wilcots, E. M., \& Liu, C. T. 2002, ApJS, 142, 161

Rafanelli, P., Violato, M., \& Baruffolo, A. 1995, AJ, 109, 1546

Rampazzo, R., \& Sulentic, J. W. 1992, A\&A, 259, 43

de Robertis, M. M., Hayhoe, K., \& Yee, H. K. C. 1998, ApJS, 115, 163

Russell, D. G. 2002, ApJ, 565, 681

Saunders, W., Sutherland, W. J., Maddox, S. J., et al. 2000, MNRAS, 317,55

Sauty, S., Casoli, F., Boselli, A., et al. 2003, A\&A, 411, 381

Schlegel, D. J., Finkbeiner, D. P., \& Davis, M. 1998, ApJ, 500, 525

Schmidt, M. 1968, ApJ, 151, 393

Schmidt, K.-H., \& Boller, T. 1992, AN, 313, 189

Schneider, S. E., Thuan, T. X., Magri, C., \& Wadiak, J. E. 1990, ApJS, 72,245

Schneider, S. E., Thuan, T. X., Mangum, J. G., \& Miller, J. M. 1992, ApJS, 81, 5

Sharina, M. E., Karachentsev, I. D., \& Tikhonov, N. A. 1999, AstL, 25,322

Sofue, Y., Tomita, A., Tutui, Y., Honma, M., \& Takeda, Y. 1998, PASJ, 50,427

Solanes, J. M., Sanchis, T., Salvador-Sole, E., Giovanelli, R., \& Haynes, M. P. 2002, AJ, 124, 2440

Stocke, J. T. 1978, AJ, 83, 348
Strauss, M. A., Huchra, J. P., Davis, M., et al. 1992, ApJS, 83, 29

Sulentic, J. W. 1976, ApJS, 32, 171

Sulentic, J. W. 1987, ApJ, 322, 605

Sulentic, J. W. 1989, AJ, 98, 2066

Sulentic, J. W., \& de Mello Rabaca, D. 1993, ApJ, 410, 520

Sulentic, J. W., \& Rabaca, C. R. 1994, ApJ, 429, 531

Stocke, J. T., Keeney, B. A., Lewis, A. D., Epps, H. W., \& Schild, R. E. 2004, AJ, 127, 1336

Swaters, R. A., van Albada, T. S., van der Hulst, J. M., \& Sancisi, R. 2002, A\&A, 390, 829

Teerikorpi, P., Bottinelli, L., Gouguenheim, L., \& Paturel, G. 1992, A\&A, 260, 17

Terry, J. N., Paturel, G., \& Ekholm, T. 2002, A\&A, 393, 57

Theureau, G., Bottinelli, L., Coudreau-Durand, N., et al. 1998, A\&AS, 130, 333

Tifft, W. G., Kirshner, R. P., Moody, J. W., \& Gregory, S. A. 1986, ApJ, 310, 75

Tully, R. B. 1988 (Cambridge University Press)

Ugryumov, A. V., Engels, D., Kniazev, A. Y., et al. 2001, A\&A, 374, 907

Uson, J. M., \& Matthews, L. D. 2003, AJ, 125, 2455

van Driel, W., Ragaigne, D., Boselli, A., Donas, J., \& Gavazzi, G. 2000, A\&AS, 144, 463

van Gorkom, J. H. 1996, in Cold Gas at High Redshift, ed. M. N. Bremer et al. (Dordrecht: Kluwer), 145

Varela, J., Moles, M., Marquez, I., et al. 2004, A\&A, 420, 873

de Vaucouleurs, G., de Vaucouleurs, A., Corwin, Jr. H. G., et al. 1991, Third Reference Catalog of Bright Galaxies (Springer Verlag: New York)

Verdes-Montenegro, L., Yun, M. S., Perea, J., del Olmo, A., \& Ho, P. T. P. 1998, ApJ, 497,89

Verdes-Montenegro, L., Yun, M. S., Williams, B. A., et al. 2001, A\&A, 377, 812

Vettolani, G., de Souza, R., \& Chincarini, G. 1986, A\&A, 154, 343

Wegner, G., Haynes, M. P., \& Giovanelli, R. 1993, AJ, 105, 1251

Whiting, A. B. 2003, ApJ, 587, 186

Willick, J. A., Bowyer, S., \& Brodie, J. P. 1990, ApJ, 355, 393

Xu, C., \& Sulentic, J. W. 1991, ApJ, 374, 407

Young, L. M., Kenney, J. D., Tacconi, L, et al. 2000, AJ, 119, 188

Young, J. S., Kenney, J. D., Tacconi, L., et al. 1986, ApJ, 311, L17

Zasov, A. V., \& Sulentic, J. W. 1994, ApJ, 430, 179

Zepf, S. E., de Carvalho, R. R., \& Ribeiro, A. L. B. 1997, ApJ, 488, L11

Zwicky, F., Herzog, E., Karpowicz, M., Kowal, C., \& Wild, P. 1961-1968, Catalogue of Galaxies and of Cluster of Galaxies (Pasadena, California Institute of Technology, CGCG) 